\title{
Efficacy of Neuromuscular Training on Pain, Balance and Function in Patients with Grade I and II Knee Osteoarthritis
}

\author{
Ganjave Pranita D and Dabholkar Twinkle
}

\begin{abstract}
Aim: To assess and compare the effect of conventional exercise program alone (conventional group) with conventional exercise program in conjunction with neuromuscular training (experimental group) in patients with knee osteoarthritis. Method: Forty patients with unilateral knee osteoarthritis were randomly assigned to two treatment groups for 12 sessions: Group A-Conventional exercises group and Group B-Experimental group.Patients in both the groups were assessed on outcome measures of knee pain, knee range, muscle strength, balance parameters, knee instability and function pre-treatment, 2 weeks and 4 weeks post treatment. Results: On between group comparisons, there were no differences in pain, knee range of motion, isometric knee and hip muscle strength and end point excursion in balance. However, there was statistically greater improvement in function and some components of balance in the experimental group when compared to conventional group. Conclusion: Hence it is beneficial to add neuromuscular training to conventional exercise program in patients with knee osteoarthritis.
\end{abstract}

\author{
Ganjave Pranita D \\ MPT \\ School of Physiotherapy \\ D.Y.Patil University, Navi Mumbai, India \\ E-mail: pranitaganjave@gmail.com \\ Dabholkar Twinkle \\ Professor \\ School of Physiotherapy \\ D.Y.Patil University, Navi Mumbai, India \\ E-mail: twinkledabholkar@ rediffmail.com
}

Key Words: Knee Osteoarthritis, Balance, Neuromuscular Training

DOI: $10.18376 / j e s p / 2017 / v 13 / i 2 / 111281$

\section{Introduction}

Osteoarthritis (OA) is a chronic degenerative disorder of multifactorial etiology. It is the second most common rheumatological problem with prevalence of $22 \%$ to $39 \%$ in India (Chopra A et al.,1997,Chopra A et al.,2001,Mahajan A et al.,2003). Knee OA has a major impact on physical functioning in a daily activity and often leads to moderate to severe limitations in participation and activity limitations among elderly. Individuals with knee OA undergo progressive loss of function and other lower limb activities. In knee osteoarthritis the intracapsular, as well as the periarticular tissues, such as ligaments, capsule, tendons and muscle are affected in the disease process. Patients with knee OA may complain of knee instability, describe as "giving way" or "buckling" of the knee during activities of daily living. The prevalence of self-reported instability has ranged from 44\% among people with knee $\mathrm{OA}$ and is correlated with reduced functional ability(Fitzgerald et al.,2004).The sensation of joint instability puts the knee joint to harmful shear forces and accelerates rate of disease progression is linked with abnormal or extreme translations of the articular surfaces (Andriacchi TP et al.,2004).It has been reported that patients with knee OA exhibit greater levels of muscle co-contraction, as a compensatory strategy for knee stabilization(Lewek MD et al.,2005),but it is an ineffective strategy for limiting knee joint instability(Schmitt LC and Rudolph KS, 2008) as the symptoms and the rate of disease progression 
are affected by combined effects of excessive shear forces and muscle co-contraction(Andriacchi TP et al.,2004).Takashi and Sharma in their review have discussed that proprioception is impaired in patients with knee OA(Takashi Nagai et al.,2007,Sharma L And Pai Y,1997). It has been established that in patients with knee OA proprioceptive acuity i.e. the awareness of joint position, kinaesthesia declines both with age and as a result of osteoarthritis (Hurley MVet al., 1997,Pai Y et al.,1997)and it may lead to reduced dynamic knee stability(Lephart SM and Riemann $\mathrm{BL}$,2002). When OA affects weight-bearing joints, it leads to decline of muscle function and as a result leads to a reduction in balance (R.S.Hinman et al.,2002). Balance is an essential component of activities of daily living. Control of balance is dependent on sensory input from the vestibular, visual and somatosensory systems. Efficient control of balance thus depends on precise sensory input and also on a timely response of strong muscles(Jones $G$ et al.,2000).Control of balance is essential in all postures and situations, both static and dynamic. Postural sway is often used as an indicator of static standing balance(Era P et al., 1985 ,Kollegger H et al.,1992) . Traditionally, Exercise therapy programs for knee OA have been impairment based and primarily target impairments associated with it i.e. muscle weakness, lower-extremity joint motion deficits. Though these programs may be useful in improving these impairments, they fail to offer the individual other challenges of balance and motor function (eg. quick stops, turns, and changes in direction, challenges to balance) that may be encountered during functional activities(GK Fitzgerald,2011). Limited researches have evaluated the effect of knee OA on dynamic balance and functional mobility (Afaf AM Shaheen et al.,2008,Khalaj N et al.,2014). Most of the earlier studies utilized force platforms and sway- meter to evaluate postural sway(Hurley MVet al., 1997, R.S.Hinman et al.,2002,Hassan B et al.,2001)and simple clinical tests like step test and functional reach test, single leg stance test (R.S.Hinman et al.,2002, Hatfield Get al.,2015)to assess dynamic balance in OA patients. Also some studies to date have utilized balance master system to assess balance in individuals with knee OA as an assessment tool (Afaf AM Shaheen et al.,2008) but the effect of KBA training on balance and postural control in OA studies is not studied. The purpose of this study was to find the effect of two different treatment protocol i.e conventional exercise program alone and conventional exercise program along with neuromuscular training (KBA) on knee pain, balance, lower extremity muscle strength, range of motion and function in patient with knee osteoarthritis. In this study we have included an objective and postural control assessment with the balance master in patients with knee osteoarthritis in addition to the other outcome parameters.

\section{Materials and Method}

This study, was conducted in D.Y. Patil Hospital and Research Centre, Nerul, Navi Mumbai. Forty patients, both male and female, suffering from with grade I and II osteoarthritis of knee joint(Kellgren JH and Lawrence JS,1957), and who were able to ambulate without any assistive devices were included in the study. Exclusion criteria were patients suffering from other type of arthritis, severe arthritis (grade III) limiting independent ambulation and activities of daily living, neurological deficit involving trunk or lower extremity and with unresolved balance disorder.The eligible patients were than randomly assigned to one of the 2 groups: Group A (Conventional Exercise Program) and Group B (Conventional Exercise Program and Neuromuscular Training). A written informed consent was obtained from the patients prior to recruitment, after explaining the benefits, risks and study procedure. The patients are assessed on the aforementioned outcome measures pre-treatment, second week and $4^{\text {th }}$ week post-treatment. The patients of both the groups attended 3 treatment sessions per week for 4 weeks, for a total of 12 treatment sessions. The Outcome measures included in our study were as follows

1) Pain assessment: Numerical pain rating scale (NPRS), where 0 corresponds to "no pain" and 10 corresponds to "worst imaginable pain" was used to measure pain (Ferrar JT,2001). 
2) Range of motion:Active knee flexion and extension range of motion (ROM) were measured in degrees using Universal(Norkin CC and White DJ,1995)

3) Knee stability: Knee outcome survey -activities of daily living scale (KOS-ADLS)were used to measure self-reported knee instability (Irrgang JJ et al.,1998).

4) Function: Western Ontario and McMaster Universities Osteoarthritis Index (WOMAC) Modified CRD Pune Version is osteoarthritis specific survey consisting of sub-scales of Pain, Stiffness, and Physical Function and a total score additive of the sub-scales was used to measure self-reported activity limitations(Arvind Chopra et al.,2004).

5) Change in condition after intervention: Global Rating of Change Scale (GROC)was used to review the overall outcome of condition with physical therapy intervention(Steven $\mathbf{J}$ Kamper et al.,2009)

6) Balance assessment:

a) Sensory impairment [postural sway as measured by modified clinical test of sensory interaction on balance (mCTSIB) test and unilateral stance (US) test]

b) motor impairment [dynamic standing as measured by limit of stability (LOS) test, and Rhythmic weight shifts (RWS) test]

These components were evaluated using Balance Master System (Neurocom System 8.6.0) (NeuroCom ${ }^{\circledR}$ International).All patients started with the assessment of static balance, which was followed by the dynamic balance test. One trial was allowed before data collection.

7) Muscle strength measurements: Isometrics strength measures in kilogram (kg) were taken for the Hip Abductors, Hip External Rotators and Quadriceps using a isokinetic Push Pull Dynamometer. Participant were instructed to push against the arm of the dynamometer with maximal resistance. Subjects were made to perform test three times and average was taken for the reading.one practice trial was given before beginning the test with 30 second rest between trials.

Exercise intervention

Group A: This group received hot packs and performed lower limb flexibility exercises prior to intervention. Strength training with weights was given as per patient tolerance without causing pain for hip abductor in side-lying, hip external rotators in sitting, quadriceps in sitting and terminal knee extension and progression was done as per the comfort of the patient. 3 sets of 10 repetitions were performed for improving strength.

Group B: In addition to the conventional treatment delivered to conventional group, one set of neuromuscular training consisting of KBA (Kinaesthesia, Balance \& Agility) Exercises and balance training on stable and unstable surface. Each set was repeated twice in single treatment session.KBA utilized walking agility exercises as summarized inTable1.This program was adapted from the guidelines given by Matthew W. Rogers et al.,2011.

Table 1. KBA agility and balance exercises

\begin{tabular}{|l|c|}
\hline Exercise & Description \\
\hline $\begin{array}{l}\text { Wedding } \\
\text { march }\end{array}$ & $\begin{array}{l}\text { Step forward and slightly to one side with leading foot, bring trailing foot } \\
\text { together with leading foot ; alternate leading foot }\end{array}$ \\
\hline $\begin{array}{l}\text { Backward } \\
\text { wedding march }\end{array}$ & As above, stepping backward \\
\hline Side stepping & $\begin{array}{l}\text { Stand with feet together, step to side with leading foot, bring trailing foot } \\
\text { back to leading foot; repeat for prescribed number of steps, then repeat in } \\
\text { opposite direction }\end{array}$ \\
\hline
\end{tabular}


Journal of Exercise Science \& Physiotherapy, Vol. 13, No. 2, 2017

ISSN: 0973-2020 (Print) $\quad \mathrm{I}_{2}$ OR Impact Factor = 5.23 UGC Approved [Journal No.7485] ISSN: 2454-6089 (online)

\begin{tabular}{|l|c|}
\hline Semi-tandem & $\begin{array}{c}\text { Walk heel-to-toe with heel landing just in front of and medial to great toe of } \\
\text { opposite foot }\end{array}$ \\
\hline Tandem walk & Advanced version of above; heel lands directly in front of opposite foot \\
\hline $\begin{array}{l}\text { Cross-over } \\
\text { walk }\end{array}$ & Walk forward bringing each foot across midline of body \\
\hline $\begin{array}{l}\text { Modified } \\
\text { grapevine }\end{array}$ & $\begin{array}{l}\text { Step to side with right foot, bring left foot behind right, step to side with } \\
\text { right, bring left in front of right; repeat for prescribed number of steps; change } \\
\text { leading foot and repeat in opposite direction. }\end{array}$ \\
\hline
\end{tabular}

KBA exercises were completed at a walking pace and progressed by adding more steps. Participants began with approximately 15 steps of each exercise and progression was targeted to a maximum of 75 steps. Three sets of mentioned seven balance exercises were performed. Additionally, static balance exercises with both feet on a stable surface with eyes open and eyes closed were performed by the participants. This was progressed to one-foot standing on stable surface for 10 seconds without losing balance. Once this was achieved, the individual was progressed to both-feet standing on the unstable surface. To further challenge balance, upper limb movements i.e. reach outs were given in all directions both on stable and unstable surface.

Data was analysed using SPSS statistical software, version 16 (SPSS), and data were checked for normality prior to analysis. P-values of less than or equal to 0.05 were considered to be significant. Since the data were not normally distributed, non-parametric tests were used. Mann-Whitney U-test was used for between group comparison and Friedman test and Wilcoxon test for post hoc comparison was performed to compare changes from baseline to four weeks for within group comparison.

Results

Forty participants (30 women, 10 men) completed the 4 weeks treatment. Participant demographic characteristics at baseline are presented in Table 2.

Table 2. Participant Characteristics at Baseline

\begin{tabular}{|l|l|l|}
\hline & Age & BMI \\
\hline Group A (Conventional) & $55.4 \pm 9.27$ & $29.17 \pm 4.597$ \\
\hline Group B (Experimental) & $52.45 \pm 8.80$ & $28.53 \pm 4.62$ \\
\hline p-value & 0.1664 & 0.6620 \\
\hline
\end{tabular}

The above data (Table.2) shows that there were no statistical differences at baseline for Age and $\mathrm{BMI}$ in both the groups which show that the groups are comparable.

Table 3. Within group analysis for Mean pain, knee range of motion, knee and hip muscle strength, WOMAC scale and KOS-ADL at pre-treatment, post 2 weeks treatment and post 4 weeks of treatment (Both groups)

\begin{tabular}{|c|c|c|c|c|c|}
\hline & & \multicolumn{4}{|c|}{ WITHIN GROUP analysis (Mean \pm SD) } \\
\hline & & $\begin{array}{c}\text { Pre- } \\
\text { treatment }\end{array}$ & $\begin{array}{l}\text { Post } 2 \text { weeks } \\
\text { of treatment }\end{array}$ & $\begin{array}{l}\text { Post } 4 \text { weeks } \\
\text { of treatment }\end{array}$ & $\begin{array}{c}\mathbf{P} \\
\text { value }\end{array}$ \\
\hline Pain at rest (NPRS) & $\begin{array}{l}\text { Group } \\
\text { A }\end{array}$ & $1.7(1.1)$ & $0.4(0.82)$ & $0.2(0.41)$ & $0.000^{*}$ \\
\hline
\end{tabular}


Journal of Exercise Science \& Physiotherapy, Vol. 13, No. 2, 2017

ISSN: $0973-2020$ (Print) I I OR Impact Factor = 5.23 UGC Approved [Journal No.7485] ISSN: 2454-6089 (online)

\begin{tabular}{|c|c|c|c|c|c|}
\hline & $\begin{array}{l}\text { Group } \\
\text { B }\end{array}$ & $1.8(1.0)$ & $0.45(0.83)$ & $0.1(0.31)$ & $0.000^{*}$ \\
\hline \multirow[t]{2}{*}{ Pain on movement } & $\begin{array}{l}\text { Group } \\
\text { A }\end{array}$ & $4.21(0.36)$ & $1.95(1.73)$ & $0.85(0.74)$ & $0.000^{*}$ \\
\hline & $\begin{array}{l}\text { Group } \\
\text { B }\end{array}$ & $4.65(1.1)$ & $2.35(1.46)$ & $0.55(0.68)$ & $0.000^{*}$ \\
\hline \multirow[t]{2}{*}{ Knee flexion } & $\begin{array}{l}\text { Group } \\
\text { A }\end{array}$ & 117.2(5.9) & $119.6(5.1)$ & $123(4.5)$ & $0.000^{*}$ \\
\hline & $\begin{array}{l}\text { Group } \\
\text { B }\end{array}$ & $116.3(6.1)$ & $119.2(5.2)$ & $122.5(6.2)$ & $0.000^{*}$ \\
\hline \multirow[t]{2}{*}{ Knee flexor strength } & $\begin{array}{l}\text { Group } \\
\text { A }\end{array}$ & $8.8(2.7)$ & $8.5(2.6)$ & $10.4(2.5)$ & $0.000^{*}$ \\
\hline & $\begin{array}{l}\text { Group } \\
\text { B }\end{array}$ & $8.6(3.4)$ & $9(3.2)$ & $10.8(2.6)$ & $0.000^{*}$ \\
\hline \multirow[t]{2}{*}{$\begin{array}{l}\text { Knee extensor } \\
\text { strength }\end{array}$} & $\begin{array}{l}\text { Group } \\
\text { A }\end{array}$ & $9.3(2.9)$ & $9.4(2.4)$ & $10.4(2.6)$ & $0.001^{*}$ \\
\hline & $\begin{array}{l}\text { Group } \\
\text { B }\end{array}$ & $8.7(3.5)$ & $9.4(3.3)$ & $10.6(3.2)$ & $0.000^{*}$ \\
\hline \multirow[t]{2}{*}{$\begin{array}{l}\text { Hip external } \\
\text { rotation strength }\end{array}$} & $\begin{array}{l}\text { Group } \\
\text { A }\end{array}$ & $10.3(3.7)$ & $10.4(4.2)$ & $11.6(3.3)$ & $0.002^{*}$ \\
\hline & $\begin{array}{l}\text { Group } \\
\text { B }\end{array}$ & $11(4)$ & $11.4(3.6)$ & $11.8(2.8)$ & $0.039 *$ \\
\hline \multirow[t]{2}{*}{$\begin{array}{l}\text { Hip abductor } \\
\text { strength }\end{array}$} & $\begin{array}{l}\text { Group } \\
\text { A }\end{array}$ & $13.5(4.2)$ & $13.3(4.2)$ & $14.1(3.3)$ & $0.024 *$ \\
\hline & $\begin{array}{l}\text { Group } \\
\text { B }\end{array}$ & $13.3(3.6)$ & $13(3.7)$ & $14.6(3.2)$ & $0.000^{*}$ \\
\hline \multirow[t]{2}{*}{$\begin{array}{l}\text { Womac CRD } \\
\text { PuneVersion }\end{array}$} & $\begin{array}{l}\text { Group } \\
\text { A }\end{array}$ & $46.1(6.5)$ & $30.3(7.5)$ & $19.5(18.1)$ & $0.000^{*}$ \\
\hline & $\begin{array}{l}\text { Group } \\
\text { B }\end{array}$ & $44.35(6.9)$ & $28.1(8.9)$ & $12.7(4.8)$ & $0.000^{*}$ \\
\hline \multirow[t]{2}{*}{ KOS-ADL } & $\begin{array}{l}\text { Group } \\
\text { A }\end{array}$ & $1.45(0.5)$ & - & $3.3(0.65)$ & $0.000^{*}$ \\
\hline & $\begin{array}{l}\text { Group } \\
\text { B }\end{array}$ & $1.75(0.6)$ & - & $3.75(0.44)$ & $0.000^{*}$ \\
\hline
\end{tabular}

*P-value $<0.05$ indicate significant difference within groups.

Table 4. Within group analysis for all the component of balance

\begin{tabular}{|l|l|l|l|l|l|}
\hline \multirow{2}{*}{} & & \multicolumn{2}{|c|}{ WITHIN GROUP analysis (Mean \pm SD) } \\
\cline { 3 - 6 } & & $\begin{array}{l}\text { Pre } \\
\text { treatment }\end{array}$ & $\begin{array}{l}\text { Post 2 weeks of } \\
\text { treatment }\end{array}$ & $\begin{array}{l}\text { Post 4 weeks of } \\
\text { treatment }\end{array}$ & $\begin{array}{l}\text { P } \\
\text { value }\end{array}$ \\
\hline \multirow{2}{*}{ Mctsib } & $\begin{array}{l}\text { Group } \\
\text { A }\end{array}$ & $0.75(0.17)$ & $0.77(0.18)$ & $0.65(0.13)$ & $0.002^{*}$ \\
\cline { 2 - 6 } & $\begin{array}{l}\text { Group } \\
\text { B }\end{array}$ & $0.73(0.18)$ & $0.78(0.24)$ & $0.54(0.1)$ & $0.000^{*}$ \\
\hline
\end{tabular}


Journal of Exercise Science \& Physiotherapy, Vol. 13, No. 2, 2017

ISSN: $0973-2020$ (Print) I I OR Impact Factor = 5.23 UGC Approved [Journal No.7485] ISSN: 2454-6089 (online)

\begin{tabular}{|c|c|c|c|c|c|}
\hline \multirow[t]{2}{*}{$\begin{array}{l}\text { Unilateral stance with } \\
\text { eyes open }\end{array}$} & $\begin{array}{l}\text { Group } \\
\text { A }\end{array}$ & $2.27(1.2)$ & $2.71(1.4)$ & $1.63(0.83)$ & 0.450 \\
\hline & $\begin{array}{l}\text { Group } \\
\text { B }\end{array}$ & $2.39(1.3)$ & $2.4(1.3)$ & $2.26(0.91)$ & $0.001 *$ \\
\hline \multirow[t]{2}{*}{$\begin{array}{l}\text { Unilateral stance with } \\
\text { eyes close }\end{array}$} & $\begin{array}{l}\text { Group } \\
\text { A }\end{array}$ & $3.5(1.8)$ & $3.49(1.8)$ & $3.33(1.1)$ & 0.949 \\
\hline & $\begin{array}{l}\text { Group } \\
\text { B }\end{array}$ & $3.36(1.3)$ & $3.31(1.3)$ & $2.27(0.1)$ & $0.002 *$ \\
\hline \multirow[t]{2}{*}{ LOS Reaction time } & $\begin{array}{l}\text { Group } \\
\text { A }\end{array}$ & $1.39(0.5)$ & $1.52(0.58)$ & $1.13(0.31)$ & $0.005^{*}$ \\
\hline & $\begin{array}{l}\text { Group } \\
\text { B }\end{array}$ & $1.33(0.59)$ & $1.38(0.42)$ & $0.83(0.19)$ & $0.000^{*}$ \\
\hline \multirow[t]{2}{*}{$\begin{array}{l}\text { LOS movement } \\
\text { velocity }\end{array}$} & $\begin{array}{l}\text { Group } \\
\text { A }\end{array}$ & $2.25(0.58)$ & $2.21(0.72)$ & $2.78(0.63)$ & $0.002 *$ \\
\hline & $\begin{array}{l}\text { Group } \\
\text { B }\end{array}$ & $2.32(0.7)$ & $2.57(0.63)$ & $3.33(0.58)$ & $0.000^{*}$ \\
\hline \multirow[t]{2}{*}{$\begin{array}{l}\text { LOS Endpoint } \\
\text { excursion }\end{array}$} & $\begin{array}{l}\text { Group } \\
\text { A }\end{array}$ & $65.6(13)$ & $67.1(12)$ & $70.8(12)$ & $0.047 *$ \\
\hline & $\begin{array}{l}\text { Group } \\
\text { B }\end{array}$ & $66.7(9.8)$ & $68.25(7.7)$ & $76.6(9)$ & $0.000^{*}$ \\
\hline \multirow[t]{2}{*}{$\begin{array}{l}\text { LOS Maximum } \\
\text { excursion }\end{array}$} & $\begin{array}{l}\text { Group } \\
\text { A }\end{array}$ & $75.9(9.4)$ & $77.1(6.7)$ & $79.9(8.5)$ & $0.008^{*}$ \\
\hline & $\begin{array}{l}\text { Group } \\
\text { B }\end{array}$ & $76.9(8.7)$ & $77.7(9.6)$ & $86.2(6.7)$ & $0.000^{*}$ \\
\hline \multirow[t]{2}{*}{$\begin{array}{l}\text { LOS Directional } \\
\text { control }\end{array}$} & $\begin{array}{l}\text { Gro } \\
\text { up A }\end{array}$ & $71.3(13)$ & $74(9.3)$ & $77(11.8)$ & $0.000^{*}$ \\
\hline & $\begin{array}{l}\text { Gro } \\
\text { up B } \\
\end{array}$ & $70(18.8)$ & $75.1(4.4)$ & $83.9(4.8)$ & $0.000^{*}$ \\
\hline \multirow[t]{2}{*}{$\begin{array}{l}\text { Rhythmic weight shift } \\
\text { Left \& Right }\end{array}$} & $\begin{array}{l}\text { Group } \\
\text { A }\end{array}$ & $79.8(4.5)$ & $80.5(4.8)$ & $82(3.5)$ & $0.049^{*}$ \\
\hline & $\begin{array}{l}\text { Group } \\
\text { B }\end{array}$ & $81.5(4.8)$ & $82.3(4.3)$ & $75(3.2)$ & $0.007 *$ \\
\hline \multirow[t]{2}{*}{$\begin{array}{l}\text { Rhythmic weight shift } \\
\text { front \& Back }\end{array}$} & $\begin{array}{l}\text { Group } \\
\text { A }\end{array}$ & $70.6(7.4)$ & $71.1(8.6)$ & $73.3(11)$ & $0.006^{*}$ \\
\hline & $\begin{array}{l}\text { Group } \\
\text { B }\end{array}$ & $73(7.9)$ & $73.3(9)$ & $79.2(6.8)$ & $0.000^{*}$ \\
\hline
\end{tabular}

For within group analysis Friedman test was used, both conventional group and experimental group showed statistically significant improvement in outcome measures like pain, knee range of motion ,isometric knee and hip muscle strength, balance (modified clinical sensory interaction on balance, limit of stability test, unilateral stance, rhythmic weight shift) and functional status (WOMAC index Modified-CRD Pune version, global rate of change scale, knee outcome survey-activities of daily living scale). However there was no improvement in balance component of sway velocity for unilateral stance with eyes opened and eyes closed in conventional group. 
Journal of Exercise Science \& Physiotherapy, Vol. 13, No. 2, 2017

ISSN: $0973-2020$ (Print) I I OR Impact Factor = 5.23 UGC Approved [Journal No.7485] ISSN: 2454-6089 (online)

Table 5. Wilcoxon signed ranks test for pain, knee range of motion, knee and hip muscle strength, WOMAC scale at pre treatment, post 2 weeks treatment and post 4 weeks of treatment (Both groups)

\begin{tabular}{|c|c|c|c|c|}
\hline \multicolumn{5}{|c|}{ WITHIN GROUP analysis (Mean \pm SD) } \\
\hline & & $\begin{array}{l}\text { Pre Rx -Post 2wk } \\
\text { of Rx }\end{array}$ & $\begin{array}{l}\text { Pre Rx- Post } \\
\text { 4wk of Rx }\end{array}$ & $\begin{array}{l}\text { Post 2wk Rx- } \\
\text { Post 4wk Rx }\end{array}$ \\
\hline & & $\mathrm{P}$ value & $\mathrm{P}$ value & $\mathrm{P}$ value \\
\hline \multirow[t]{2}{*}{ Pain at rest } & Group A & $.000 *$ & $.000 *$ & $.046^{*}$ \\
\hline & Group B & $.000 *$ & $.000 *$ & $.038 *$ \\
\hline \multirow[t]{2}{*}{ Pain on movement } & Group A & $.000 *$ & $.000 *$ & $.004 *$ \\
\hline & Group B & $.000 *$ & $.000^{*}$ & $.000^{*}$ \\
\hline \multirow[t]{2}{*}{ Knee flexion } & Group A & $.002 *$ & $.000^{*}$ & $.001 *$ \\
\hline & Group B & $.009 *$ & $.000 *$ & $.016^{*}$ \\
\hline \multirow[t]{2}{*}{ knee flexor strength } & Group A & .180 & $.000 *$ & $.000 *$ \\
\hline & Group B & .206 & $.001 *$ & $.002 *$ \\
\hline \multirow{2}{*}{$\begin{array}{ll}\text { Knee } & \text { extensor } \\
\text { strength } & \end{array}$} & Group A & $.008 *$ & $.000 *$ & $.005 *$ \\
\hline & Group B & $.008 *$ & $.000 *$ & $.003 *$ \\
\hline \multirow{2}{*}{$\begin{array}{l}\text { Hip external rotation } \\
\text { Strength }\end{array}$} & Group A & .543 & $.033^{*}$ & .073 \\
\hline & Group B & .480 & $.008^{*}$ & $.013 *$ \\
\hline \multirow{2}{*}{$\begin{array}{ll}\text { Hip } & \text { abductor } \\
\text { strength }\end{array}$} & Group A & .317 & .083 & $.021 *$ \\
\hline & Group B & .157 & $.003^{*}$ & $.000^{*}$ \\
\hline \multirow{2}{*}{ Womac scale } & Group A & $.000^{*}$ & $.000^{*}$ & $.000 *$ \\
\hline & Group B & $.000 *$ & $.000^{*}$ & $.000 *$ \\
\hline
\end{tabular}

Table 6. Wilcoxon signed ranks test(p value)for within group comparison of all the component of balance at pre-treatment, post 2 weeks treatment and post 4 weeks of treatment

\begin{tabular}{|c|c|c|c|c|}
\hline \multicolumn{5}{|c|}{ WITHIN GROUP analysis (Mean \pm SD) } \\
\hline & & $\begin{array}{l}\text { Pre Rx -Post } \\
\text { 2wk of Rx }\end{array}$ & $\begin{array}{l}\text { Pre Rx- Post } \\
\text { 4wk of Rx }\end{array}$ & $\begin{array}{l}\text { Post 2wk Rx- } \\
\text { Post 4wk Rx }\end{array}$ \\
\hline & & $\mathrm{P}$ value & $\mathrm{P}$ value & $\mathrm{P}$ value \\
\hline \multirow[t]{2}{*}{ Mctsib } & Group A & .601 & $.000 *$ & $.019 *$ \\
\hline & Group B & .071 & $.001 *$ & $.000 *$ \\
\hline \multirow{2}{*}{$\begin{array}{l}\text { Unilateral stance with } \\
\text { eyes open }\end{array}$} & Group A & .779 & .732 & .737 \\
\hline & Group B & .126 & $.001 *$ & $.002 *$ \\
\hline \multirow{2}{*}{$\begin{array}{l}\text { Unilateral stance with } \\
\text { eyes close }\end{array}$} & Group A & .904 & .628 & .763 \\
\hline & Group B & .926 & $.002 *$ & $.005 *$ \\
\hline \multirow[t]{2}{*}{ LOS Reaction time } & Group A & .601 & $.028 *$ & $.019 *$ \\
\hline & Group B & .673 & $.001 *$ & $.000 *$ \\
\hline \multirow{2}{*}{$\begin{array}{l}\text { LOS movement } \\
\text { velocity }\end{array}$} & Group A & .888 & $.001 *$ & $.001 *$ \\
\hline & Group B & .135 & $.000 *$ & $.000 *$ \\
\hline \multirow{2}{*}{$\begin{array}{ll}\text { LOS } & \text { Endpoint } \\
\text { excursion } & \end{array}$} & Group A & .322 & $.002 *$ & .089 \\
\hline & Group B & .545 & $.000^{*}$ & $.001 *$ \\
\hline
\end{tabular}


Journal of Exercise Science \& Physiotherapy, Vol. 13, No. 2, 2017

ISSN: $0973-2020$ (Print) $\quad \mathrm{I}_{2}$ OR Impact Factor = 5.23 UGC Approved [Journal No.7485] ISSN: 2454-6089 (online)

\begin{tabular}{|l|l|l|l|l|}
\hline \multirow{2}{*}{$\begin{array}{l}\text { LOS Maximum } \\
\text { excursion }\end{array}$} & Group A & .640 & $.008^{*}$ & .064 \\
\cline { 2 - 5 } $\begin{array}{l}\text { LOS Directional } \\
\text { control }\end{array}$ & Group B & .556 & $.000^{*}$ & $.000^{*}$ \\
\hline \multirow{2}{*}{$\begin{array}{l}\text { Rhythmic weight } \\
\text { shift Left \& Right }\end{array}$} & Group B & .098 & $.000^{*}$ & $.029^{*}$ \\
\hline \multirow{2}{*}{$\begin{array}{l}\text { Rhythmic weight } \\
\text { shifts front \& back }\end{array}$} & Group A & .743 & $.000^{*}$ & $.000^{*}$ \\
\cline { 2 - 5 } & Group B & .614 & $.016^{*}$ & .093 \\
\cline { 2 - 5 } & Group A & .940 & $.002^{*}$ & $.011^{*}$ \\
\hline
\end{tabular}

For within group post hoc analysis (Table 5\&6)of pain (NRPS), knee range of motion, knee and hip muscle strength, WOMAC scale, KOS-ADL,GROC and all the component of balance, Wilcoxon test was used. On statistical comparison of baseline score with post 2 weeks score, there was statistical significant improvement in pain,knee range of motion, and function (WOMAC), but nonsignificant changes in knee and hip muscle strength and balance parameters in both the groups. When post 2 weeks and 4 weeks of treatment scores were compared there was statistically significant reduction in pain score and statistically significant improvement in all the component (i.e. knee range of motion, knee and hip muscle strength, WOMAC scale, KOS-ADL), with except that there was no significant change in hip external rotation strength in conventional group. There were significant improvements in all balance parameters in the experimental group, but in the conventional group showed improvements only in 4 out 10 balance parameters namely modified CTSIB, Limit of stability reaction time, movement velocity, and directional control. When comparing baseline scores with post 4 weeks scores, there was statistical significant improvement in pain, knee range of motion, hip and knee muscle strength, and function (WOMAC) in both the groups, with the exception of non-significant improvement in hip abductor strength in the conventional group. There were significant improvements in all balance parameters in the experimental group, with the exception of non-significant improvement in unilateral stance balance parameter in the conventional group.

Table 7. Between group analysis for pain, knee range of motion, knee and hip muscle strength, WOMAC scale, KOS-ADL,GROC at pre-treatment, post 2 weeks treatment and post 4 weeks of treatment (Both group)

\begin{tabular}{|c|c|c|c|c|c|c|c|c|c|}
\hline \multicolumn{10}{|c|}{ BETWEEN GROUP analysis (Mean \pm SD) } \\
\hline \multirow{2}{*}{$\begin{array}{l}\text { Outcome } \\
\text { Measures }\end{array}$} & \multicolumn{3}{|c|}{ Pre -treatment } & \multicolumn{3}{|c|}{$\begin{array}{lcc}\text { Post } 2 & \text { weeks } & \text { of } \\
\text { treatment }\end{array}$} & \multicolumn{3}{|c|}{$\begin{array}{lccc}\begin{array}{l}\text { Post } \quad 4 \\
\text { treatment }\end{array} & \text { weeks } & \text { of } \\
\end{array}$} \\
\hline & $\begin{array}{l}\text { Group } \\
\text { A }\end{array}$ & $\begin{array}{l}\text { Group } \\
\text { B }\end{array}$ & $\begin{array}{l}\mathrm{P} \\
\text { Value }\end{array}$ & $\begin{array}{l}\text { Group } \\
\text { A }\end{array}$ & $\begin{array}{l}\text { Group } \\
\text { B }\end{array}$ & $\begin{array}{l}\mathrm{P} \\
\text { value }\end{array}$ & $\begin{array}{l}\text { Group } \\
\text { A }\end{array}$ & $\begin{array}{l}\text { Group } \\
\text { B }\end{array}$ & $\begin{array}{l}\mathrm{P} \\
\text { value }\end{array}$ \\
\hline Pain at rest & $\begin{array}{l}1.7 \\
(1.1)\end{array}$ & $\begin{array}{l}1.8 \\
(1.0)\end{array}$ & 0.808 & $\begin{array}{l}0.4 \\
(0.82)\end{array}$ & $\begin{array}{l}0.45 \\
(0.83)\end{array}$ & 0.766 & $\begin{array}{l}0.2 \\
(0.41)\end{array}$ & $\begin{array}{l}0.1 \\
(0.31)\end{array}$ & 0.382 \\
\hline $\begin{array}{l}\text { Pain on } \\
\text { movement }\end{array}$ & $\begin{array}{l}4.2 \\
(1.36)\end{array}$ & $\begin{array}{l}4.65 \\
(1.1)\end{array}$ & 0.219 & $\begin{array}{l}1.95 \\
(1.73)\end{array}$ & $\begin{array}{l}2.35 \\
(1.46)\end{array}$ & 0.205 & $\begin{array}{l}0.85 \\
(0.74)\end{array}$ & $\begin{array}{l}0.55 \\
(0.68)\end{array}$ & 0.185 \\
\hline Knee flexion & $\begin{array}{l}117.2 \\
(5.9)\end{array}$ & $\begin{array}{l}116.3 \\
(6.1)\end{array}$ & 0.407 & $\begin{array}{l}119.6 \\
(5.1)\end{array}$ & $\begin{array}{l}119.2 \\
(5.2)\end{array}$ & 0.742 & $\begin{array}{l}123 \\
(4.5)\end{array}$ & $\begin{array}{l}122.5 \\
(6.2)\end{array}$ & 0.625 \\
\hline $\begin{array}{l}\text { Knee flexor } \\
\text { strength }\end{array}$ & $\begin{array}{l}8.8 \\
(2.7)\end{array}$ & $\begin{array}{l}8.6 \\
(3.4)\end{array}$ & 0.603 & $\begin{array}{l}8.5 \\
(2.6)\end{array}$ & $9(3.2)$ & 0.415 & $\begin{array}{l}10.4 \\
(2.5)\end{array}$ & $\begin{array}{l}10.8 \\
(2.6)\end{array}$ & 0.709 \\
\hline
\end{tabular}


Journal of Exercise Science \& Physiotherapy, Vol. 13, No. 2, 2017

ISSN: 0973-2020 (Print) $\quad \mathrm{I}_{2}$ OR Impact Factor = 5.23 UGC Approved [Journal No.7485] ISSN: 2454-6089 (online)

\begin{tabular}{|c|c|c|c|c|c|c|c|c|c|}
\hline $\begin{array}{l}\text { Knee } \\
\text { extensor } \\
\text { strength }\end{array}$ & $\begin{array}{l}9.3 \\
(2.9)\end{array}$ & $\begin{array}{l}8.7 \\
(3.5)\end{array}$ & 0.228 & $\begin{array}{l}9.4 \\
(2.4)\end{array}$ & $\begin{array}{l}9.4 \\
(3.3)\end{array}$ & 0.942 & $\begin{array}{l}10.4 \\
(2.6)\end{array}$ & $\begin{array}{l}10.6 \\
(3.2)\end{array}$ & 0.966 \\
\hline $\begin{array}{l}\text { Hip external } \\
\text { rotation } \\
\text { strength }\end{array}$ & $\begin{array}{l}10.3 \\
(3.7)\end{array}$ & $11(4)$ & 0.749 & $\begin{array}{l}10.4 \\
(4.2)\end{array}$ & $\begin{array}{l}11.4 \\
(3.6)\end{array}$ & 0.832 & $\begin{array}{l}11.6 \\
(3.3)\end{array}$ & $\begin{array}{l}11.8 \\
(2.8)\end{array}$ & 0.989 \\
\hline $\begin{array}{l}\text { Hip abductor } \\
\text { strength }\end{array}$ & $\begin{array}{l}13.5 \\
(4.2)\end{array}$ & $\begin{array}{l}13.3 \\
(3.6) \\
\end{array}$ & 0.783 & $\begin{array}{l}13.3 \\
(4.2)\end{array}$ & $\begin{array}{l}13 \\
(3.7)\end{array}$ & 0.741 & $\begin{array}{l}14.1 \\
(3.3)\end{array}$ & $\begin{array}{l}14.6 \\
(3.2)\end{array}$ & 0.626 \\
\hline $\begin{array}{l}\text { Womac Pune } \\
\text { Version }\end{array}$ & $\begin{array}{l}46.1 \\
(6.5) \\
\end{array}$ & $\begin{array}{l}44.4 \\
(6.9) \\
\end{array}$ & 0.328 & $\begin{array}{l}30.3 \\
(7.5) \\
\end{array}$ & $\begin{array}{l}28.1 \\
(8.9)\end{array}$ & 0.569 & $\begin{array}{l}19.5 \\
(8.1) \\
\end{array}$ & $\begin{array}{l}12.7 \\
(4.8)\end{array}$ & $0.007 *$ \\
\hline KOS-ADL & $\begin{array}{l}1.45 \\
(0.5)\end{array}$ & $\begin{array}{l}1.75 \\
(0.6)\end{array}$ & 0.090 & - & - & - & $\begin{array}{l}3.3 \\
(0.65)\end{array}$ & $\begin{array}{l}3.8 \\
(0.44)\end{array}$ & $0.020 *$ \\
\hline GROC & - & - & - & - & - & - & $1.2(1)$ & $\begin{array}{l}1.9 \\
(0.96)\end{array}$ & $0.004 *$ \\
\hline
\end{tabular}

Table 8. Between group analysis for all the component of balance at pre treatment, post 2 weeks treatment and post 4 weeks of treatment (Both group)

\begin{tabular}{|c|c|c|c|c|c|c|c|c|c|}
\hline \multicolumn{10}{|c|}{ BETWEEN GROUP analysis (Mean \pm SD) } \\
\hline \multirow{2}{*}{$\begin{array}{l}\text { Outcome } \\
\text { Measures }\end{array}$} & \multicolumn{3}{|c|}{ Pre -treatment } & \multicolumn{3}{|c|}{$\begin{array}{c}\begin{array}{c}\text { Post 2 weeks of } \\
\text { treatment }\end{array} \\
\end{array}$} & \multicolumn{3}{|c|}{$\begin{array}{c}\text { Post } 4 \text { weeks of } \\
\text { treatment }\end{array}$} \\
\hline & $\begin{array}{l}\text { Group } \\
\text { A }\end{array}$ & $\begin{array}{l}\text { Group } \\
\text { B }\end{array}$ & $\begin{array}{l}\mathrm{P} \\
\text { Value }\end{array}$ & $\begin{array}{l}\text { Group } \\
\text { A }\end{array}$ & $\begin{array}{l}\text { Group } \\
\text { B }\end{array}$ & $\begin{array}{l}\mathrm{P} \\
\text { value }\end{array}$ & $\begin{array}{l}\text { Group } \\
\text { A }\end{array}$ & $\begin{array}{l}\text { Group } \\
\text { B }\end{array}$ & $\begin{array}{l}\mathrm{P} \\
\text { Value } \\
\end{array}$ \\
\hline mCTSIB & $\begin{array}{l}0.75 \\
(0.17)\end{array}$ & $\begin{array}{l}0.73 \\
(0.18)\end{array}$ & 0.107 & $\begin{array}{l}0.77 \\
(0.18)\end{array}$ & $\begin{array}{l}0.78 \\
(0.24)\end{array}$ & 0.752 & $\begin{array}{l}0.65 \\
(0.13)\end{array}$ & $\begin{array}{l}0.54 \\
(0.1) \\
\end{array}$ & $0.010^{*}$ \\
\hline $\begin{array}{l}\text { Unilateral } \\
\text { stance with } \\
\text { eyes open }\end{array}$ & $\begin{array}{l}2.27 \\
(1.2)\end{array}$ & $\begin{array}{l}2.39 \\
(1.3)\end{array}$ & 0.635 & $\begin{array}{l}2.71 \\
(1.4)\end{array}$ & $\begin{array}{l}2.4 \\
(1.3)\end{array}$ & 0.524 & $\begin{array}{l}1.63 \\
(0.8)\end{array}$ & $\begin{array}{l}2.26 \\
(0.9)\end{array}$ & $0.017 *$ \\
\hline $\begin{array}{l}\text { Unilateral } \\
\text { stance with } \\
\text { eyes close }\end{array}$ & $\begin{array}{l}3.5 \\
(1.8)\end{array}$ & $\begin{array}{l}3.36 \\
(1.3)\end{array}$ & 0.829 & $\begin{array}{l}3.49 \\
(1.8)\end{array}$ & $\begin{array}{l}3.31 \\
(1.3)\end{array}$ & 0.978 & $\begin{array}{l}3.33 \\
(1.1)\end{array}$ & $\begin{array}{l}2.27 \\
(0.1)\end{array}$ & $0.004 *$ \\
\hline $\begin{array}{l}\text { LOS Reaction } \\
\text { Time }\end{array}$ & $\begin{array}{l}1.39 \\
(0.5)\end{array}$ & $\begin{array}{l}1.33 \\
(0.59)\end{array}$ & 0.860 & $\begin{array}{l}1.52 \\
(0.58)\end{array}$ & $\begin{array}{l}1.38 \\
(0.42)\end{array}$ & 0.655 & $\begin{array}{l}1.13 \\
(0.31)\end{array}$ & $\begin{array}{l}0.83 \\
(0.19)\end{array}$ & $0.001 *$ \\
\hline $\begin{array}{l}\text { LOS } \\
\text { movement } \\
\text { velocity }\end{array}$ & $\begin{array}{l}2.25 \\
(0.58)\end{array}$ & $\begin{array}{l}2.32 \\
(0.7)\end{array}$ & 0.349 & $\begin{array}{l}2.21 \\
(0.72)\end{array}$ & $\begin{array}{l}2.57 \\
(0.63)\end{array}$ & $0.030^{*}$ & $\begin{array}{l}2.78 \\
(0.63)\end{array}$ & $\begin{array}{l}3.33 \\
(0.58)\end{array}$ & $0.006^{*}$ \\
\hline $\begin{array}{l}\text { LOS } \\
\text { Endpoint } \\
\text { excursion }\end{array}$ & $\begin{array}{l}65.6 \\
(13)\end{array}$ & $\begin{array}{l}66.7 \\
(9.8)\end{array}$ & 1.000 & $\begin{array}{l}67.1 \\
(12)\end{array}$ & $\begin{array}{l}68.25 \\
(7.7)\end{array}$ & 0.914 & $\begin{array}{l}70.8 \\
(12)\end{array}$ & $\begin{array}{l}76.6 \\
(9)\end{array}$ & 0.085 \\
\hline $\begin{array}{l}\text { LOS } \\
\text { Maximum } \\
\text { excursion }\end{array}$ & $\begin{array}{l}75.9 \\
(9.4)\end{array}$ & $\begin{array}{l}76.9 \\
(8.7)\end{array}$ & 0.714 & $\begin{array}{l}77.1 \\
(6.7)\end{array}$ & $\begin{array}{l}77.7 \\
(9.6)\end{array}$ & 0.786 & $\begin{array}{l}79.9 \\
(8.5)\end{array}$ & $\begin{array}{l}86.2 \\
(6.7)\end{array}$ & $0.049 *$ \\
\hline $\begin{array}{l}\text { LOS } \\
\text { Directional }\end{array}$ & $\begin{array}{l}71.3 \\
(13)\end{array}$ & $\begin{array}{l}70 \\
(18.8)\end{array}$ & 0.914 & $\begin{array}{l}74 \\
(9.3)\end{array}$ & $\begin{array}{l}75.1 \\
(4.4)\end{array}$ & 0.776 & $\begin{array}{l}77 \\
(11.8)\end{array}$ & $\begin{array}{l}83.9 \\
(4.8)\end{array}$ & $0.030 *$ \\
\hline
\end{tabular}


Journal of Exercise Science \& Physiotherapy, Vol. 13, No. 2, 2017

ISSN: 0973-2020 (Print) $\quad \mathrm{I}_{2} \mathrm{OR}$ Impact Factor $=5.23$ UGC Approved [Journal No.7485]] ISSN: 2454-6089 (online)

\begin{tabular}{|c|c|c|c|c|c|c|c|c|c|}
\hline control & & & & & & & & & \\
\hline $\begin{array}{l}\text { Rhythmic } \\
\text { weight shift } \\
\text { Lt \& Rt }\end{array}$ & $\begin{array}{l}79.8 \\
(4.5)\end{array}$ & $\begin{array}{l}81.5 \\
(4.8)\end{array}$ & 0.206 & $\begin{array}{l}80.5 \\
(4.8)\end{array}$ & $\begin{array}{l}82.3 \\
(4.3)\end{array}$ & 0.260 & $\begin{array}{l}82 \\
(3.5)\end{array}$ & $\begin{array}{l}75 \\
(3.2)\end{array}$ & $0.002 *$ \\
\hline $\begin{array}{l}\text { Rhythmic } \\
\text { weight shift } \\
\text { front\& Back }\end{array}$ & $\begin{array}{l}70.6 \\
(7.4)\end{array}$ & $\begin{array}{l}73 \\
(7.9)\end{array}$ & 0.343 & $\begin{array}{l}71.1 \\
(8.6)\end{array}$ & $\begin{array}{l}73.3 \\
(11)\end{array}$ & 0.401 & $\begin{array}{l}73.3 \\
(11)\end{array}$ & $\begin{array}{l}79.2 \\
(6.8)\end{array}$ & $0.034 *$ \\
\hline
\end{tabular}

For between group statistical comparisons of all outcome measures, Mann-Whitney U-test was used. (Table.7 \& 8). When both the groups were compared at baseline there was no statistically significant difference in all outcome measures, which show that the groups are comparable.The result of present study shows that on between group comparison(Table.7\&8) at baseline and post 2 weeks of treatment, there was no difference in all the outcome measures. However, post 2 weeks of treatment there was difference in movement velocity (limit of stability) in balance in experimental group. When both the groups were compared post 4 weeks of treatment there was no statistically significant difference in pain (numerical pain rating score), knee range of motion, isometric knee and hip muscle strength and end point excursion in balance (limit of stability test) in both the groups. However, experimental group showed greater improvement in function (WOMAC index Modified-CRD pune version, GROC scale, self-reported instability on Knee outcome surveyactivities of daily living scale and some components of balance (modified clinical sensory interaction on balance, limit of stability, unilateral stance and rhythmic weight shift) than conventional group.

\section{Discussion}

This study aimed to compare the efficacy of only conventional exercises with conventional exercise program along with neuromuscular training on knee pain, balance, lower extremity muscle strength, range of motion and function, in patients with knee osteoarthritis. The results of study indicates that 4 weeks of treatment consisting of conventional exercise program or conventional exercise program along with neuromuscular training (KBA) both led to reduced pain, improved range of motion, lower extremity muscle strength, knee instability, function and balance in both the groups. The post treatment reduction of pain could be the result of exercise training in both the groups as exercises stimulates mechanoreceptor in muscle (A beta fibres) which leads to suppression of impulses through pain gate mechanism, which also applies when exercises are administered in patients with OA. The results of our study are in accordance with the findings of Robert Topp et al.,2002in supporting that the dynamic or resistance training reduces knee joint pain in patients with knee OA. Also hot packs given prior to intervention can be attributed to reduction in pain as heat is often used in conjunction with exercises to treat chronic musculoskeletal problems(Val Robertson et al.)Pain relieving effects of heat are mainly reflex, possibly an activation of pain gate mechanism. Heat application reduces level of muscle spasm usually associated with it (Val Robertson et al.). Improvement in the knee range of motion could be due administration of stretching exercises to both groups(Dean C et al.,1990,Kieran O'Sullivan etal.,2009). Overall exercises improves the proprioceptive mechanism of joint leading to more normal excursion of the joint and contributes to increased range of motion by inhibiting pain through continued A-beta firing during these activities and help in controlling pain. Lori et a.,2011 reported that rehabilitation should focus on quadriceps and hip strengthening and additional attention to the external rotators along with gluteus medius may be useful. A similar strength protocol was administered to both the groups. In our study both the groups showed improvement in quadriceps, hamstrings and hip muscle strength post treatment.Improvement in strength can be due to reduction ofpain, as pain may cause voluntary 
inhibition leading to an inability of the quadriceps muscle function properly and prevents it from being activated leading to imbalance between muscle groups(Riann Met al.,2013) Use of resistance training in rehabilitation and conditioning program has an impact on all systems of the body. Adaptations to overload create changes in muscle fibre resulting in hypertrophy of the muscle fibres and thus become increasingly important adaptation that accounts for strength gain in muscle(Roitman,JL,2001). Although KBA training was administered only in the experimental group, even the conventional group showed improvements in balance parameters. It has been proven that strength training can lead to improvements in balance(SP Messier et al.,2000)and function(Jun Iwamoto et al., 2011).Lee and Park have reported that improvement in the strength of the lower extremities can enhance balance(Park S,2010). On between group comparisons there were similar improvements in pain, knee range of motion and knee and hip muscle strength and balance parameters in both groups, with the exception of higher improvements in $6 / 10$ balance parameters in experimental group . The probable reasons for improvement in pain, range of motion and strength in both the groups are discussed earlier. The greater improvement seen in modified CTSIB, limit of stability, unilateral stance and rhythmic weight shifts in experimental group which was given neuromuscular training could be the effect of improved knee proprioception and knee stability through neuromuscular training(KBA)(Mathews W.Rogers et al., 2011,Demirhan D et al.,2005) as studies have shown that KBA techniques improves dynamic joint stability using a series of physical activities such as agility and balance exercises to activate, challenge, and adapt the nervous system's proprioceptors to maintain balance and to improve activities of daily living function(Mathews W.Rogers et al., 2011).Exercises using an unstable base of support had a positive effect on improving balance ability and decreasing pain (Kyung Kim et al.,2016) Also it could be the effect of repetitions of task aimed at improving motor control at knee joint. The ultimate goal of proprioceptive training is to improve or restore sensory and/or sensorimotor function. Neuromuscular control mediated through proprioception of joint takes into account three distinct levels of motor control activation within the CNS. Reflexes at the spinal level mediate movement pattern that are received from higher levels of the nervous system. This action provides for reflex joint stabilization during conditions of abnormal stress about the articulation. The second level of motor control, located within the brainstem, receives input from joint mechanoreceptors, vestibular centres and visual input from the eyes to maintain posture and balance of the body. The highest level of CNS function provides cognitive awareness of body positions and movement in which motor commands are initiated for voluntary movements.(Lephart S.M et al.,1997). Incorporating these three levels of motor control activities to address proprioceptive deficiencies encourages maximum afferent discharge to the respective CNS level. Enhancing motor function at the brainstem level and at the cortical level to stimulate the conversion of conscious and unconscious motor programming can be achieved by performing postural and balance activities, both with and without visual input. In our study balance training with eyes open and eyes closed on firm and nonconforming surface and balance training with sets of KBA exercises was given in the experimental group. Closed chain exercises, which cause joint approximation, stimulate mechanoreceptors in muscles and in and around joints to enhance sensory input for the control of movement (Kisner C, Colby L A.,2007) The balance exercise training protocol of our study was conducted in the weight bearing position (i.e. during standing or walking). Kinesthetic and proprioception training are such type of activity that can enhance this function(Lephart, S.M et al.,1997). Also neuromuscular training stimulates proprioceptive input, enhances muscular joint stabilization and postural function(Aman, J.E et al.,2015).Thus the result of the present study can be attributed to the effect of neuromuscular training (KBA) which effectively activates the brainstem and cortical level of motor control. Also the results of our study complement those of Sekir and Gur.,2005in supporting the potential of KBA as a superior treatment option. Strengthening exercises alone have effects on 
improving functional outcomes and joint stability in patient with OA (Pelland, L et al.,2004, Oliveiraet al.,2012,Knoop, J et al.,2013) this can be attributed to improvement in function in both the groups as measured on WOMAC index, global rating of change scale as both the groups received same strength training protocol. Also it is proven that lower extremity strengthening enhances the balance in patients with knee OA (SP Messier et al.,2000) which contributes to increase participation of an individual in functional activities. Incorporating neuromuscular training has been shown to improve function(GK Fitzgerald et al.,2011) and reduce knee instability in knee osteoarthritis patients(MW Rogers et al.,2011). Hence the greater improvement in functional outcome scores [WOMAC, subscale of difficulty on WOMAC scale $(\mathrm{P}=0.020)$ and GROC] and knee instability in experimental group could be attributed to improvement in postural control and proprioceptive accuracy through neuromuscular training(Amal F Ahmed.,2011). KBA techniques improves dynamic joint stability using number of physical activities which challenge neuromuscular system to maintain balance and coordination (Matthew W et al.,2011).

Further studies can be carried out on patients with Grade III osteoarthritis. Perturbation exercises can be incorporated in neuromuscular training program.

\section{Conclusion}

Based on our study results, we conclude that although conventional exercise program is effective in reducing knee pain, and increasing lower extremity muscle strength and range of motion, adding neuromuscular training (KBA) along with conventional exercise program in rehabilitation leads to higher improvement on balance and function in patient with knee grade I and II osteoarthritis.

\section{References}

Ahmed, A.F., 2011. Effect of sensorimotor training on balance in elderly patients with knee osteoarthritis. Journal of Advanced Research, 2(4):305-311.

Aman, J.E., Elangovan, N., Yeh, I.L. and Konczak, J., 2014. The effectiveness of proprioceptive training for improving motor function: a systematic review. Frontiers in human neuroscience, 8 .

Andriacchi, T.P., Mündermann, A., Smith, R.L., Alexander, E.J., Dyrby, C.O. and Koo, S., 2004. A framework for the in vivo pathomechanics of osteoarthritis at the knee. Annals of biomedical engineering, 32(3):447-457.

Bolgla, L.A., Malone, T.R., Umberger, B.R. and Uhl, T.L., 2011. Comparison of hip and knee strength and neuromuscular activity in subjects with and without patellofemoral pain syndrome. The international journal of sports physical therapy, 6(4):285.

Chopra A, Patil J, Bilampelly V, Relwane J, Tandle HS.,2001.Prevalence of rheumatic disease in rural population in Western India: A WHO-ILAR-COPCORD study. $J$ Assoc Physicians India,49:240-46.

Chopra, A., Lavin, P., Patwardhan, B. and Chitre, D., 2004. A 32-week randomized, placebo-controlled clinical evaluation of RA-11, an Ayurvedic drug, on osteoarthritis of the knees. JCR: Journal of Clinical Rheumatology, 10(5):236-245.

Chopra, A., Patil, J., Billampelly, V., Relwani, J. and Tandale, H.S., 1997. The Bhigwan (India) COPCORD: methodology and first information report. APLAR J Rheumatol, 1:145-54.

Diracoglu, D., Aydin, R., Baskent, A. and Celik, A., 2005. Effects of kinesthesia and balance exercises in knee osteoarthritis. JCR: Journal of Clinical Rheumatology, 11(6):303-310.

Era, P. and Heikkinen, E., 1985. Postural sway during standing and unexpected disturbance of balance in random samples of men of different ages. Journal of Gerontology, 40(3):287-295.

Farrar, J.T., Young, J.P., LaMoreaux, L., Werth, J.L. and Poole, R.M., 2001. Clinical importance of changes in chronic pain intensity measured on an 11-point numerical pain rating scale. Pain, 94(2):149-158.

Fitzgerald, G.K., Piva, S.R. and Irrgang, J.J., 2004. Reports of joint instability in knee osteoarthritis: its prevalence and relationship to physical function. Arthritis Care \& Research, 51(6):941-946.

Fitzgerald, G.K., Piva, S.R., Gil, A.B., Wisniewski, S.R., Oddis, C.V. and Irrgang, J.J., 2011. Agility and perturbation training techniques in exercise therapy for reducing pain and improving function in people with knee osteoarthritis: a randomized clinical trial. Physical therapy, 91(4):452 
Hassan, B.S., Mockett, S. and Doherty, M., 2001. Static postural sway, proprioception, and maximal voluntary quadriceps contraction in patients with knee osteoarthritis and normal control subjects. Annals of the rheumatic diseases, 60(6):612-618.

Hatfield, G.L., Morrison, A., Wenman, M., Hammond, C.A. and Hunt, M.A., 2016. Clinical tests of standing balance in the knee osteoarthritis population: systematic review and meta-analysis. Physical therapy, 96(3):324-337.

Hinman, R.S., Bennell, K.L., Metcalf, B.R. and Crossley, K.M., 2002. Balance impairments in individuals with symptomatic knee osteoarthritis: a comparison with matched controls using clinical tests. Rheumatology, 41(12):1388-1394

Hurley, M.V., Scott, D.L., Rees, J. and Newham, D.J., 1997. Sensorimotor changes and functional performance in patients with knee osteoarthritis. Annals of the rheumatic diseases, 56(11):641-648.

Irrgang, J.J., Snyder-Mackler, L., Wainner, R.S., Fu, F.H. and HARNER, C.D., 1998. Development of a patient-reported measure of function of the knee. J Bone Joint Surg Am, 80(8):1132-45.

Iwamoto, J., Sato, Y., Takeda, T. and Matsumoto, H., 2011. Effectiveness of exercise for osteoarthritis of the knee: A review of the literature. World J Orthop, 2(5):37-42.

Kamper, S.J., Maher, C.G. and Mackay, G., 2009. Global rating of change scales: a review of strengths and weaknesses and considerations for design. Journal of Manual \& Manipulative Therapy, 17(3):163170.

Kandel, E.R., Schwartz, J.H. and Jessell, T.M. eds., 2000. Principles of neural science (Vol. 4, pp. 12271246). New York: McGraw-hill.

Kellgren, J.H. and Lawrence, J.S., 1957. Radiological assessment of osteo-arthrosis. Annals of the rheumatic diseases, 16(4):494-502.

Khalaj, N., Osman, N.A.A., Mokhtar, A.H., Mehdikhani, M. and Abas, W.A.B.W., 2014. Balance and risk of fall in individuals with bilateral mild and moderate knee osteoarthritis. PloS one, 9(3):e92270.

Kim, K., Lee, H.Y. and Lim, S.J., 2016. Effects of increased standing balance on pain in patients with knee osteoarthritis. Journal of physical therapy science, 28(1),87-89.

Kisner C, Colby L A.,2007.Therapeutic exercise foundation and techniques, 5 th ed.:178

Knoop, J., Dekker, J., Van der Leeden, M., Van der Esch, M., Thorstensson, C.A., Gerritsen, M., Voorneman, R.E., Peter, W.F., de Rooij, M., Romviel, S. and Lems, W.F., 2013. Knee joint stabilization therapy in patients with osteoarthritis of the knee: a randomized, controlled trial. Osteoarthritis and Cartilage, 21(8):1025-1034.

Kollegger, H., Baumgartner, C., Wöber, C., Oder, W. and Deecke, L., 1992. Spontaneous body sway as a function of sex, age, and vision: posturographic study in 30 healthy adults. European neurology, 32(5):253-259

Lephart, S.M., Pincivero, D.M., Giraido, J.L. and Fu, F.H., 1997. The role of proprioception in the management and rehabilitation of athletic injuries. The American journal of sports medicine, 25(1):130-137.

Lewek, M.D., Ramsey, D.K., Snyder-Mackler, L. and Rudolph, K.S., 2005. Knee stabilization in patients with medial compartment knee osteoarthritis. Arthritis \& Rheumatism, 52(9):2845-2853.

Mahajan, A., Jasrotia, D.S., Manhas, A.S. and Jamwal, S.S., 2003. Prevalence of major rheumatic disorders in Jammu. JK Science, 5:63-66.

Messier, S.P., Royer, T.D., Craven, T.E., O'toole, M.L., Burns, R. and Ettinger, W.H., 2000. Long-term exercise and its effect on balance in older, osteoarthritic adults: Results from the Fitness, Arthritis, and Seniors Trial (FAST). Journal of the American Geriatrics Society, 48(2):131-138.

Nagai, T., Sell, T.C. and Lephart, S.M., 2007. Effect of Age and Osteoarthritis on Knee Proprioception. US Musculoskeletal Review,69-70.

NeuroCom ${ }^{\circledR}$ International, In.

Norkin, C.C. and White, D.J., 2016. Measurement of joint motion: a guide to goniometry. FA Davis.

Oliveira, A.M.I.D., Peccin, M.S., Silva, K.N.G.D., Teixeira, L.E.P.D.P. and Trevisani, V.F.M., 2012. Impact of exercise on the functional capacity and pain of patients with knee osteoarthritis: a randomized clinical trial. Revistabrasileira de reumatologia, 52(6):876-882. 


\section{Journal of Exercise Science \& Physiotherapy, Vol. 13, No. 2, 2017 \\ ISSN: $0973-2020$ (Print) I I OR Impact Factor = 5.23 UGC Approved [Journal No.7485] ISSN: 2454-6089 (online)}

O'Sullivan, K., Murray, E. and Sainsbury, D., 2009. The effect of warm-up, static stretching and dynamic stretching on hamstring flexibility in previously injured subjects. BMC musculoskeletal disorders, 10(1):37.

Pai, Y.C., Rymer, W.Z., Chang, R.W. and Sharma, L., 1997. Effect of age and osteoarthritis on knee proprioception. Arthritis \& Rheumatism, 40(12):2260-2265.

Palmieri-Smith, R.M., Villwock, M., Downie, B., Hecht, G. and Zernicke, R., 2013. Pain and effusion and quadriceps activation and strength. Journal of athletic training, 48(2):186-191.

Park, S.M., 2010. Meta-analysis of the interventions for preventing falls by the elderly in the eight countries: comparison between aged 70's and 80's. J Korean Gerontol, 30:49-63.

Pelland, L., Brosseau, L., Wells, G., MacLeay, L., Lambert, J., Lamothe, C., Robinson, V. and Tugwell, P., 2004. Efficacy of strengthening exercises for osteoarthritis (part I): a meta-analysis. Physical therapy reviews, 9(2):77-108.

Riemann, B.L. and Lephart, S.M., 2002. The sensorimotor system, part II: the role of proprioception in motor control and functional joint stability. Journal of athletic training, 37(1):80-84

Rogers, M.W., Tamulevicius, N., Coetsee, M.F., Curry, B.F. and Semple, S.J., 2011. Knee osteoarthritis and the efficacy of kinesthesia, balance \& agility exercise training: a pilot study. International journal of exercise science, 4(2):124-132.

Rogers, M.W., Tamulevicius, N., Semple, S.J., Coetsee, M.F. and Curry, B.F., 2011. Comparison of clinicbased versus home-based balance and agility training for the symptoms of knee osteo-arthritis. South African Journal of Sports Medicine, 23(3):80-83.

Roitman ,JL(ed) 2001.ACSM's Resource Manual for guidelines for exercise testing and prescription, 4 ed. Philadelphia: Lippincott Williams \& Wilkins.

Schmitt, L.C. and Rudolph, K.S., 2008. Muscle stabilization strategies in people with medial knee osteoarthritis: the effect of instability. Journal of Orthopaedic Research, 26(9):1180-1185.

Sekir, U. and Gür, H., 2005. A multi-station proprioceptive exercise program in patients with bilateral knee osteoarthrosis: functional capacity, pain and sensoriomotor function. A randomized controlled trial. Journal of sports science \& medicine, 4(4):590.

Shaheen, A.A. and Ayad, K., 2008. Impact of chronic osteoarthritis of knee joint on postural stability and functional mobility in women. Bull. Fac. Ph. Th. Cairo Univ, 13(1):319-333.

Sharma, L. and Pai, Y.C., 1997. Impaired proprioception and osteoarthritis. Current opinion in rheumatology, 9(3):253-258

Taylor, D.C., Dalton JR, J.D., Seaber, A.V. and Garrett JR, W.E., 1990. Viscoelastic properties of muscletendon units: the biomechanical effects of stretching. The American journal of sports medicine, 18(3):300-309.

Topp, R., Woolley, S., Hornyak, J., Khuder, S. and Kahaleh, B., 2002. The effect of dynamic versus isometric resistance training on pain and functioning among adults with osteoarthritis of the knee. Archives of physical medicine and rehabilitation, 83(9):1187-1195.

Val Robertson, PhD, Alex Ward, PhD, John Low, BA(Hons), FCSP, DipTP and Ann Reed, BA, MCSP, DipTP . (ed). Electrotherapy Explained, 4th Edition Principles and Practice :elsevier;333-334. 